\title{
Artificial Neural Networks Models with Fuzziness and Chaos Phenomena
}

\author{
Algis GARLIAUSKAS \\ Vilnius Gediminas Technical University \\ Saulètekio 11, LT-2054 Vilnius, Lithuania \\ e-mail: galgis@ktl.mii.lt
}

Received: January 2003

\begin{abstract}
We consider a generalized model of neural network with a fuzziness and chaos. The origin of the fuzzy signals lies in complex biochemical and electrical processes of the synapse and dendrite membrane excitation and the inhibition mechanism. The mathematical operations included into fuzzy neural network modeling are: the scalar product between inputs of layers and synaptic weights is replaced by a fuzzy logic multiplication, the sum of products changes to the fuzzy logic sums, and the operators such as supremum, maximum, and minimum are presented for a fuzzy description. The algorithm of varying membership functions, built basing on a backpropagation paradigm and a method of fuzzy neural optimization, has been considered. Both fuzzy properties and a chaos phenomenon are analyzed basing upon experimental computations.
\end{abstract}

Key words: Fuzzy neural network, membership, fuzzy backpropagation, chaos.

\section{Introduction}

The preliminary analysis of strong nonlinear feedbacks including the cases of bistability showed the appearance of new neural network properties, such as bifurcations, quasiperiodic oscillations, chaos presence in (van der Mass et al., 1990; Gupta and Rao, 1994; Fukai and Shiino, 1992; Garliauskas and Gupta, 1995; Garliauskas, 1997 and others). The outstanding works of Zadeh, 1965, 1968, Gupta, 1989, Kaufmann and Gupta, 1991 are linked with neural network fuzzy properties which are wide used in our search. The bistability of a dendrite membrane state considering dynamic processes in the synapsedendrite complex is related with the saddle point stability area because these processes are not sufficiently determined under weak fluctuations which causes the situations of fuzzy logic interpretation. The model of a neuron as well as fuzzy logic ideas are expressed mathematically in the most general form in the works (Gupta, 1989; 1992). The origin of the fuzzy signals lies in complex biochemical and electrical processes of the synapse and the dendrite membrane that can be classified into two groups: the first group is associated with the impact of mediators when transferring the potassium, sodium, and chlorine ions in the synapse and the dendrite membrane in the cases of excitation, inhibition, and silent inhibition; the second group is connected with a nonlinear or bistable 
mathematical representation of electrical processes causing the formation of higher order fuzzy sets. As a result, due to the fact that learning images are frequently fuzzy distorted, e.g., fingerprints in criminal cases, images from space, handwritten characters, etc., the creation of fuzzy algorithms is a perspective way. Therefore in the investigation was expedient to do a further generalization of fuzzy artificial neural networks (ANN) basing on fuzzy properties.

\section{Fuzzy Backpropagation ANN Algorithm}

\subsection{Fuzzy Statement in ANN.}

The main theoretical background of the fuzzy logic was set by L.A. Zadeh (1965). The first fuzzy algorithms for the decision-making have been developed in his and joint with R.E. Bellman issues (Zadeh, 1968; Zadeh and Bellman, 1970). A notion of a graded membership became the fundamental characteristic of information arising from a human cognitive process. A man always uses simple linguistic constructions such as small, big, large, many, medium, substantial, approximate, etc. There are no crisp boundaries among different cognitive classes or sets. Such a fuzzy statement is valid completely for neural systems in the brain-making mechanism as a human reasoning capability (Gupta, 1992; Zimmermann, 1991; Gupta and Rao, 1994). Here, contrary to the simple binary set theory, the fuzzy set theory describes events that either do or do not occur. This is partially similar as in the probability theory with measuring of an event occurring by probability, only in the fuzzy logic a relative graded

membership is used as a function of thinking and cognitive processes. The fuzziness of cognitive classes or images is not estimated by grades of membership appearing between a fuzzy and nonfuzzy situation. Note that a mathematical technique dealing with the description of fuzziness is quite different from that used in the probability theory.

\subsection{Fuzzy ANN Architecture}

The architecture of fuzzy ANN, as usual, is in related with imprecise values of input data and ill-defined actual states inside neuronal structures. The main sources of uncertainty and fuzziness in neural networks are as follows:

(i) a fuzziness that takes through a fuzzifier on the input data or forward from imprecise information, voiced signals, images, initial conditions of control systems, etc.;

(ii) fluctuations in the biochemical mechanism of synapses through activations of transmitters, number of sodium, potassium, and chlorine channels during bursts of sustained activation;

(iii) ill-defined somatic relations inside of the neuron through membrane channels and their changed plasticity;

(iv) inner chaotic phenomena of ANN defined by bifurcation parameters and their behavior when by interchanging between two frequency mode processes.

The fuzzy NN block diagram is presented in Fig. 1. 


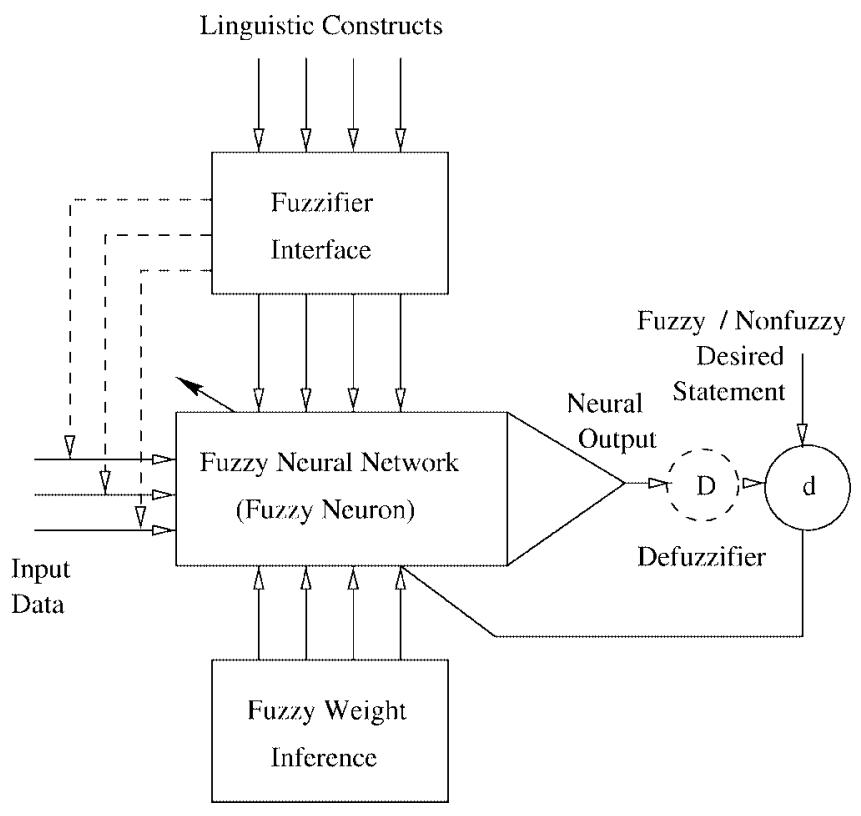

Fig. 1. Fuzzy NN block-diagram.

There is a block of a fuzzifier interface fuzzifying boundaries of input data or entering linguistic constructs which are joined with the input data set. Another block of fuzziness sources can be the fuzzy weight one according to item (ii) and presented as a fuzzy weight inference structure. An important implication possesses the fuzzy processes inside an individual neuron and neural network in general according to items (iii) and (iv). The output after the fuzzy NN mapping must be defuzzified in the defuzzifier block to compare with a desired statement and to define the global error to be minimized through the feedback adjunction procedures. For a desired fuzzy desired statement, the Hemming or Euclidean distance can be used instead of the defuzzifier.

\subsection{Fuzzy NN Algorithmic Structure}

We build a fuzzy NN algorithmic structure close to the fuzzy backpropagation paradigm because it involves a feedforward characterized as a static process and a feedback as a dynamic one (Fig. 2). In the feedforward trace, the fuzzy logic or fuzzy arithmetic operations can be used, and a defuzzifier elaboration in order to match up to a nonfuzzy desired statement or to a fuzzy desired one. After evaluating of the global error, the feedback operations are to be set in dynamics. Inside of these algorithmic procedures, the different fuzzy sources must be mapped and an iterative procedure can be continued. 


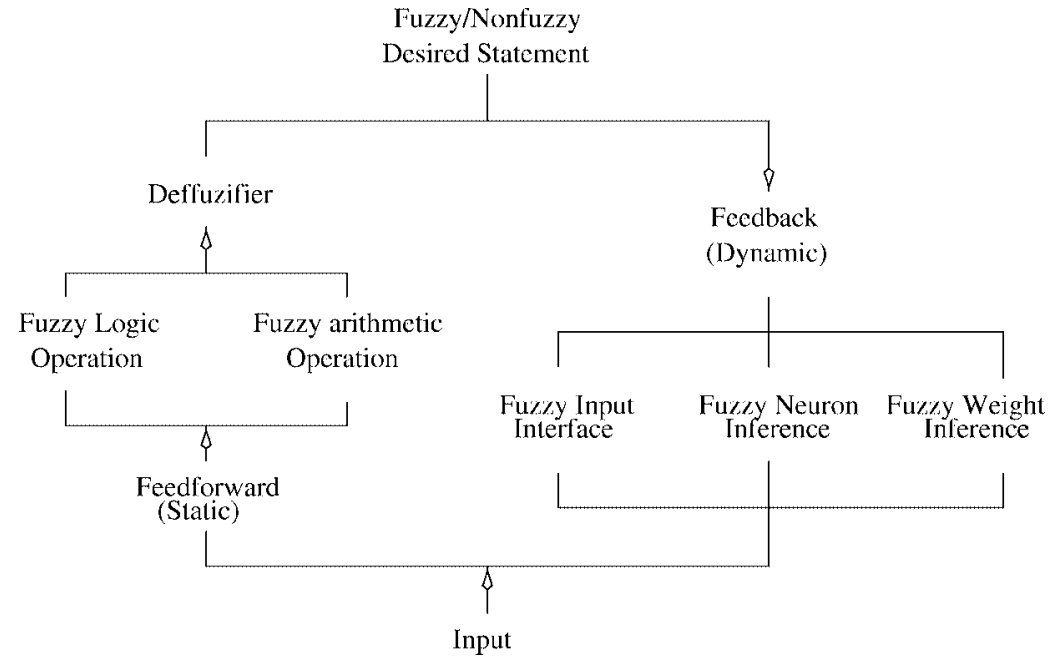

Fig. 2. Fuzzy NN algorithmic structure.

\subsection{Mathematical Fuzzy Modeling on Backpropagation}

The multi-layered neural networks (MLNN) are most propagated neural systems which mimic certain appropriate brain-style portions well and very frequently apply in different technical areas, such as pattern/image recognition, system control and identification, biometric systems and many others. Any MLNN consists of an input layer, one or more hidden layers and an output layer. It is schematically presented in Fig. 3 where a feedback trace is pointed out too.

If to note only a feedforward trace, the input-output mapping can be mathematically represented as follows

$$
\mathbf{Y}(t)=N_{n}\left[N_{n-1} \ldots\left[N_{2}\left[N_{1}\left[\mathbf{X}(t) \in R^{n}\right]\right] \ldots\right] \in R^{m}\right.
$$

where $R^{n}$ is an $n$-dimensional space of inputs data, and $R^{m}$ is an $m$-dimensional space of output state parameters.

Further, consider three-layered NN (output, 2 - hidden, and input layers) and a training algorithm when input data and weight sets are fuzzified. Such a fuzzy NN deals with a mapping of the input fuzzy sets defined as $A \in R^{n}$ which are characterized by a membership relation $\mu_{L}: A \rightarrow M$ labelled by linguistic variables from the set $L$. Here $M$

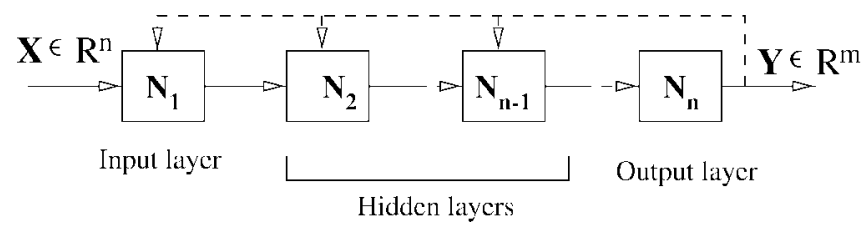

Fig. 3. Multi-layered neural network structure. 
is a membership space and it is a one-dimensional space in the interval $[0,1]$. Note, $n$ advance, that the mathematical operations, included in fuzzy NN modeling, are:

(i) the scalar product between inputs of layers and synaptic weights is replaced by fuzzy logic multiplication;

(ii) the sum of products (i) changes according to the fuzzy logic sum;

(iii) the operators as supremum, maximum, minimum will be used for a fuzzy description.

The three-layered NN was composed of layers with inputs $\widehat{x}_{k}^{(\cdot)}$ and memberships $\mu_{A}(\widehat{x})$, outputs $x_{k}^{(\cdot)}$ and $\mu_{B}(x)$, weights $w_{l k}$ and $\mu_{C}(w)$, thresholds $Q_{k}^{(\cdot)}$ and $\mu_{D}(Q)$, bias $x_{0}^{(\cdot)} \in A$. We also assume that all input data are summarized in the inputs of the first layer. There are appropriate sets $A, B, C$, and $D$ of space $R$. We include new symbolic notations of the logic conjunction $\sum^{\wedge}$ and logic disjuntion $\sum^{V}$, where symbol $\sum$ is not an algebraic sum and it is not a simple sum and expresses only as a short definition of a sequence of logic operations.

Then, for the outputs of the input layer we obtain

$$
\mu_{A}\left(x_{s}^{(0)}\right)=\left|\varphi^{(-1)}\left(x_{s}^{(0)}\right) \wedge \mu_{A}\left(\widehat{x}_{s}^{(0)}\right)-\mu_{D}\left(Q_{s}^{(0)}\right)\right|, \quad s=0,1,2, \ldots, n,
$$

where at $s=0$ it means a nonfuzzy bias with $\mu_{A}\left(\widehat{x}_{0}^{(0)}\right)=1$.

For the output of the first hidden layer in the origin, we have to define the memberships of input data of layers as follows

$$
\mu_{A}\left(\widehat{x}_{l}^{(1)}\right)=\sum_{s}^{V} \mu_{A}\left(x_{s}^{(0)}\right) \wedge \mu_{C}\left(w_{l s}^{(1)}\right), \quad l, s=0,1,2, \ldots, n,
$$

and of the output one

$$
\mu_{A}\left(x_{l}^{(1)}\right)=\left|\varphi^{-1}\left(x_{l}^{(1)}\right) \wedge \mu_{A}\left(\widehat{x}_{l}^{(1)}\right)-\mu_{D}\left(Q_{l}^{(1)}\right)\right|,
$$

for inputs of the second hidden layer

$$
\mu_{A}\left(\widehat{x}_{k}^{(2)}\right)=\sum_{l}^{V} \mu_{A}\left(x_{l}^{(1)}\right) \wedge \mu_{C}\left(w_{k l}^{(2)}\right), \quad k, l=0,1,2, \ldots, n,
$$

and

$$
\mu_{A}\left(x_{k}^{(2)}\right)=\left|\varphi^{-1}\left(x_{k}^{(2)}\right) \wedge \mu_{A}\left(\widehat{x}_{k}^{(2)}\right)-\mu_{D}\left(Q_{k}^{(2)}\right)\right|,
$$

for inputs of the output layer of NN

$$
\mu_{A}\left(\widehat{x}_{j}^{(3)}\right)=\sum_{k}^{V} \mu_{A}\left(x_{k}^{(2)}\right) \wedge \mu_{C}\left(w_{j k}^{(3)}\right), \quad j=0,1,2, \ldots, m, k=0,1,2 \ldots, n,
$$


and

$$
\mu_{A}\left(x_{j}^{(3)}\right)=\left|\varphi^{-1}\left(x_{j}^{(3)}\right) \wedge \mu_{A}\left(\widehat{x}_{j}^{(3)}\right)-\mu_{D}\left(Q_{j}^{(3)}\right)\right| .
$$

In (2.1), (2.3), (2.5), and (2.7) supremum is taken over the set of points $\varphi^{-1}\left(x_{(\cdot)}^{(\cdot)}\right)$ in $\widehat{\mathbf{X}} \in R^{n}$, i.e., $\varphi: \widehat{\mathbf{X}} \rightarrow \widehat{\mathbf{X}}$. The $\varphi^{-1}\left(x_{(\cdot)}^{(\cdot)}\right)$ is an inversed sigmoid unipolar function

$$
\widehat{x}_{(\cdot)}^{(\cdot)}=\frac{1}{\ln e} \ln \frac{x_{(\cdot)}^{(\cdot)}}{1-x_{(\cdot)}^{(\cdot)}},
$$

where $e$ is the base of a natural algorithm.

Let us set a desirable vector $\mathbf{X}^{(\mathbf{0})}$ in the output of $\mathrm{NN}$ which is nonfuzzy, i.e., $\mu\left(x_{j}^{(0)}\right)=1$ for all the components of the output vector $\mathbf{X}^{(\mathbf{0})}$. Sometimes the desired vector can be used as a fuzzy one, $\mu\left(x_{j}^{(0)}\right) \neq 1$ from $\mathrm{M}[0.1]$.

Then the minimum of the square Euclidean as a criterion of optimizing can be taken as follows

$$
e^{2}=\sum_{j=1}^{m}\left[\mu\left(x_{j}^{(0)}\right)-\mu\left(x_{j}^{(3)}\right)\right]^{2} .
$$

If according to Fig. 1 we take in to account, the presence of a defuzzifier, the output of NN can be represented by the defuzziness procedure, where basing upon the vector representation we get

$$
\mathbf{Y}\left(\mathbf{X}^{(3)}\right)=\frac{\sum_{j}^{V} \mathbf{Y}\left(\mathbf{X}^{(3)}\right) \wedge \mu_{j}\left(\mathbf{X}^{(3)}\right),}{\sum_{j}^{V} \mu_{j}\left(\mathbf{X}^{(3)}\right)}, j=0,1,2, \ldots, m .
$$

After the defuzzification, we can use the usual global error criterion:

(i) with a defuzzifier

$$
e=\frac{1}{2} \sum_{j=1}^{m}\left(y_{j}^{(0)}-y_{j}\right)^{2},
$$

(ii) without a defuzzifier

$$
E=\frac{1}{2} \sum_{j=1}^{m}\left(x_{j}^{(0)}-x_{j}^{(3)}\right)^{2} .
$$

Let us consider the main backpropagation algorithm of MLNN based on a nonfuzzy paradigm and the known dynamic learning rule of weight changes for any layer at discrete time

$$
\mathrm{w}(t+1)=\mathrm{w}(t)+\Delta \mathrm{w}(t)
$$


and

$$
\Delta \mathrm{w}(t)=-\gamma \frac{\partial E}{\partial \mathrm{w}}
$$

where $\gamma$ is the learning rate and $\mathrm{w}(t)$ is the synaptic strength over an instant in time $t$. Then the changes of the synaptic strengths in all the layers of MLNN will be the following

$$
\Delta \mathrm{w}_{j, k}^{(3)}=-\gamma \frac{\partial E}{\partial w_{j, k}^{(3)}}=-\gamma \frac{\partial E}{\partial x_{j}^{(3)}} \frac{\partial x_{j}^{(3)}}{\partial \widehat{x}_{k}^{(3)}} \frac{\partial \widehat{x}_{k}^{(3)}}{\partial w_{j, k}^{(3)}}=\gamma\left(x_{j}^{(0)}-x_{j}^{(3)}\right) x_{j}^{(3)}\left(1-x_{j}^{(3)}\right) x_{k}^{(2)}
$$

where $k=0,1,2, \ldots, n, \mathrm{w}_{j, k}^{(3)}$ is the weight of bias at which $\widehat{x}_{j}^{(3)}=1$.

Besides, $x_{j}^{(3)}=\varphi\left(\widehat{x}_{j}^{(3)}\right), \widehat{x}_{j}^{(3)}=\sum_{k=0}^{n} x_{k}^{(2)} \mathrm{w}_{j k}^{(3)}$, and the local error denote the

$$
e_{j}^{(3)}=\left(x_{j}^{(0)}-x_{j}^{(3)}\right) x_{j}^{(3)}\left(1-x_{j}^{(3)}\right)
$$

Analogously, using (9) we obtain

$$
\Delta \mathrm{w}_{k, l}^{(2)}=\gamma \frac{\partial E}{\partial w_{k, l}^{(2)}}=\gamma x_{k}^{(2)}\left(1-x_{k}^{(2)}\right) x_{l}^{(1)} \sum_{j=1}^{m} e_{j}^{(2)} \mathrm{w}_{j, k}^{(2)}=x_{m}^{(1)} e_{m}^{(2)},
$$

for the second layer, where

$$
e_{m}^{(2)}=x_{k}^{(2)}\left(1-x_{k}^{(2)}\right) \sum_{j=1}^{m} e_{j}^{(3)} \mathrm{w}_{j k}^{(3)}
$$

and

$$
\Delta \mathrm{w}_{l, s}^{(1)}=\gamma \frac{\partial E}{\partial w_{l, s}^{(1)}}=\gamma x_{l}^{(1)}\left(1-x_{l}^{(1)}\right) x_{s}^{(0)} \sum_{k=1}^{n} e_{k}^{(2)} \mathrm{w}_{k, l}^{(2)}=x_{s}^{(1)} e_{s}^{(1)},
$$

where

$$
e_{s}^{(1)}=x_{l}^{(1)}\left(1-x_{l}^{(1)}\right) \sum_{k=1}^{n} e_{k}^{(2)} \mathrm{w}_{k, l}^{(2)} .
$$

Now we return to the fuzziness of presentation of an error-based learning algorithm. Note that temporal evolution of set $C$ of weights by (7.1) does not take place, that is, the set of weight memberships is independent of time. Then a membership equation of (7.1) becomes as follows

$$
\mu_{D}\left(\mathrm{w}(t+1)=\mu_{D}(\mathrm{w}(t)) \wedge \mu_{D}(\Delta \mathrm{w}(\mathrm{t}))\right.
$$


Next consider only equations (8)-(11), e.g., (10). Then the changes of weights in the fuzzy representation will be

$$
\mu_{D}\left(\Delta \mathrm{w}_{\mathrm{k}, 1}^{(2)}\right)=\gamma\left[\mu_{\mathrm{A}}\left(\mathrm{x}_{\mathrm{m}}^{(1)}\right) \wedge \mu_{\mathrm{F}}\left(\mathrm{e}_{\mathrm{m}}^{(2)}\right)\right],
$$

where $\gamma<1$ now stands for a weighting coefficient and $\mu_{F}\left(e_{m}^{(2)}\right)$ is the membership of a fuzzy local error of the set $F \subset R$. It will be as follows

$$
\mu_{F}\left(e_{m}^{(2)}\right)=\mu_{A}\left(x_{k}^{(2)}\right) \wedge\left[1-\mu_{A}\left(x_{k}^{(2)}\right)\right] \wedge \sum_{j}^{V} \mu_{F}\left(e_{j}^{(3)}\right) \wedge \mu_{C}\left(\mathrm{w}_{\mathrm{j}, \mathrm{k}}^{(3)}\right)
$$

and, according to (9),

$$
\mu_{F}\left(e_{j}^{(3)}\right)=\left[\mu_{A}\left(x_{j}^{(0)}\right)-\mu_{A}\left(x_{j}^{(3)}\right)\right] \wedge \mu_{A}\left(x_{j}^{(3)}\right) \wedge\left(1-\mu_{A}\left(x_{j}^{(3)}\right)\right) .
$$

In this manner it is easy to transform all equations (8)-(11) to a fuzzy space and to perform the required calculations according to the fuzzy NN algorithmic structure represented in Fig. 2. Thus, the postulates of the fuzzy paradigm in NN was presented by the whole devolopment of the fuzzy backpropagation algorithm to the multi-layered NN which was used in the joint with chaos framework in NN in Section 3.

\section{Chaos Joint with Fuzzy Neural Networks}

\subsection{Presumptions of Chaos in NN.}

A neural network chaotic phenomenon is strong by connected with a better understanding of signal and pattern recognition under noise or fuzzy conditions as well as the information transform and transmission. The amount of information transmission in chaotic dynamic systems was calculated by Matsumoto and Tsuda (1987; 1988). It has been proved that a chaotic neural network has an ability of an effective transmission of any information received from outside. Fukai and Shiino (1992) discovered a chaos route via Hopf bifurcations in the neural network model with time delay. The asymmetric neural networks in terms of a mean field theory found chaos through an appearance of homoclinic orbits (Tsuda, 1992). The nonlinear dynamics and chaos theory problems in NN are discussed in (Chapeau-Blondeau and Chauvet, 1992; Garliauskas and Shaw, 1977; Garliauskas, 1999, and others). Most of the techniques that have been developed so far can be only applied in relatively simple systems. All investigators try to use dynamics theory more or less in nonlinear and far from equilibrium states of physical systems as a certain mechanism for explaining brain information processing of higher animals.

In dynamic systems, chaos is characterized by many factors. One of them is conditions of the appearence of a strange attractor. The second one is based on a pseudo-orbit tracing property which under appropriate conditions can become an approximation of some true orbit with a sufficient accuracy. The strange attractor which is of non-uniform character 
stems from dynamic intermittent systems. The crucial property of intermittent chaotic behavior is interchange between two frequency modes: high and low ones. Below we present the model which has a slow equation (low frequency) and a fast equation (high frequency). We also add a condition of fuzziness to them referring to input data and synaptic strengths.

\subsection{Chaotic Model of NN with Fuzziness}

We have performed the simulation based on a chaotic NN paradigm with a fuzziness. The model includes two frequency modes (van der Maas et al., 1990) with an essential modification introducing biologically plausable so called $N$-shaped synaptic couplings and a certainty condition that defines the fuzziness. The model is organized in the following way. The Cohen-Grossberg equations (Cohen and Grossberg, 1983), after simplifying and introducing a nonlinear $N$-shaped postsynaptic potential function, become fast (high frequency) equations discretized in time. It is written for the second (hidden) layer as follows

$$
\hat{x}_{i}^{(2)}(t+1)=\left(1-\alpha_{x}\right) \hat{x}_{i}^{(2)}(t)+\beta \rho_{i}\left(x_{i}^{(1)}\right),
$$

where

$$
\rho_{i}\left(\hat{x}_{i}^{(1)}\right)=\sum_{j=0}^{n} \mathrm{w}_{\mathrm{ij}}^{(1)} \rho_{j}\left(x_{j}\right),
$$

$\alpha_{x}$ is the decay parameter of potentials, $\beta$ is the excitatory rate, $\rho_{j}\left(x_{j}\right)$ is the $N$-shaped synaptic function further simplified as $\rho(x)$ because of its independence of time and neuron type.

Fuzzy equivalent equations are presented

$$
\mu_{A}\left(\hat{x}_{i}^{(2)}(t+1)\right)=\omega_{x} \mu_{A}\left(\hat{x}_{i}^{(2)}(t)\right) V \beta \mu_{A}\left(\rho\left(x_{i}^{(1)}\right),\right.
$$

where

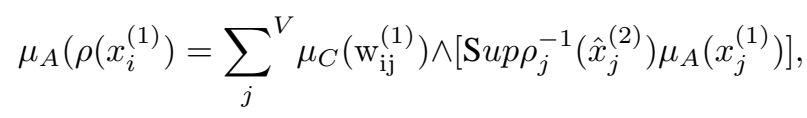

and $\omega_{x}=1-\alpha \leqslant 1$ and $\beta \leqslant 1$ are weight coefficients.

The synaptic function $\rho(x)$ reflects the mutual activity between presynaptic and postsynaptic potentials as a result of complex synapse-dendrite activation. This activation, in most cases, is defined by a restricted $N$-shaped current-voltage dendritic membrane relation (Garliauskas and Shaw, 1977; Garliauskas, 1998) that possesses two stable points and one unstable point.

Delta equations considered as slow (low frequency) ones were presented in such a way:

$$
\mathrm{w}_{\mathrm{ij}}^{(1)}(\mathrm{k}+1)=\omega_{\mathrm{w}} \mathrm{w}_{\mathrm{ij}}^{(1)}(\mathrm{k})+\eta \hat{x}_{i}^{(2)}(k) x_{j}^{(1)}(k),
$$


where $k$ is a recursion step. The fuzzy equivalent is

$$
\mu_{C}\left(\mathrm{w}_{\mathrm{ij}}^{(1)}(\mathrm{k}+1)\right)=\omega_{\mathrm{w}} \mu_{C}\left(\mathrm{w}_{\mathrm{ij}}^{(1)}(\mathrm{k})\right) V \eta\left[\mu _ { A } \left(\hat{x}_{i}(k) \wedge \mu_{A}\left(x_{j}(k)\right] .\right.\right.
$$

The solution of equations $(17.1,17.2)$ by an iterative process upto the steady state inside of the solution of slow equations $(18.1,18.2)$ amounting to 200 cycles makes a decision with a fuzziness and chaos presented in Section 4.

\section{Experimental Simulation}

The modeling experiment has been done on simple cases of an MLNN architecture: 3$2-1$, i.e., three input, two hidden and one output elements of the neuronal scheme with nonlinear functions of synaptic connections and neuronal activities as well as fuzzy conditions. The model and its developing realization, based on two-modes discrete nonlinear equations (16.2), (17.2), (18.2), were carried out under the initial conditions

$$
\widehat{I_{i}}=\{(1 \mid 0.7),(-1 \mid 0.1),(1 \mid 0.9)\},
$$

$i=1,2,3$ for three input neurons as well as synaptic weights as follows:

(i) between the input layer and a hidden one

$$
\begin{aligned}
\mathrm{w}_{\mathrm{ij}}^{(1)}=\{( & \left.\mathrm{w}_{11}^{(1)} \mid 0.9\right),\left(\mathrm{w}_{12}^{(1)} \mid 1.0\right),\left(\mathrm{w}_{13}^{(1)} \mid 0.1\right),\left(\mathrm{w}_{21}^{(1)} \mid 0.5\right), \\
& \left.\left(\mathrm{w}_{22}^{(1)} \mid 0.7\right),\left(\mathrm{w}_{23}^{(1)} \mid 0.1\right)\right\} ;
\end{aligned}
$$

(ii) between the output layer and a hidden one

$$
\mathrm{w}_{\mathrm{ij}}^{(2)}=\left\{\left(\mathrm{w}_{11}^{(2)} \mid 0.7\right),\left(\mathrm{w}_{12}^{(2)} \mid 0.3\right),\left(\mathrm{w}_{13}^{(2)} \mid 0.9\right)\right\} .
$$

The results of modeling in more extended situations are demonstrated in the diagrams of Figs. 4 and 5.

Two nonlinear functions are given: one as a unipolar sigmoidal (chaos diagram, Fig. 4) or a bipolar one (chaos diagram, Fig. 5) and the second as a weight multiplied by the $N$-shaped function. The modeling was caried out with the same parameters as above. It should be noted: first, the intermittent stable, period-doubling, unstable, and chaotic processes change place from the range with lower values of the bifurcation parameter $(\eta=17.0)$ to the range with higher ones $(\eta=34.0)$ (Fig. 4); second, the processes become richer and more complex. The diagram (Fig. 5) shows that the processes do not possess explicitly distinct period-doubling behaviour but they possess very many windows with stable point areas at $\eta=13.0 \div 17.5,19.2,24.5 \div 27.5$. Only at higher values of $\eta=29.5$ the chaotic regime is continued uninterrupted. The evolution of dynamic processes, given the bipolar sigmoidal activation function as a hyperbolic tangent and the $N$-shaped form in neuronal couplings, become less distinct and more chaotic in the general sense. 


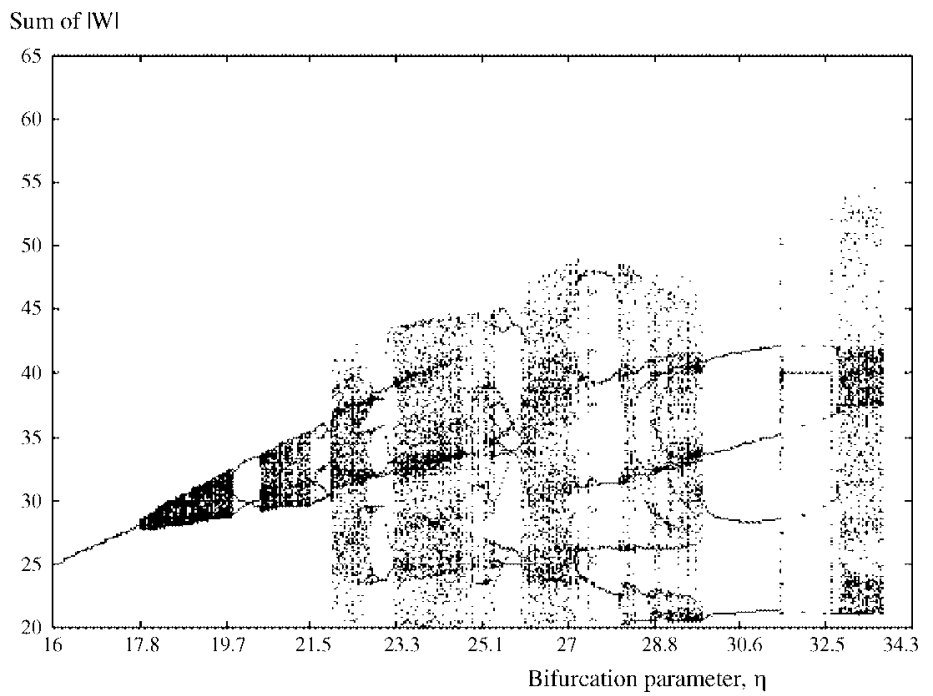

Fig. 4. Bifurcation and the chaos diagram for NN with a unipolar sigmoidal of the activation function and $n$-shaped synaptic couplings. There are $\Delta x=0.0001$ and $\Delta \eta=0.03$.

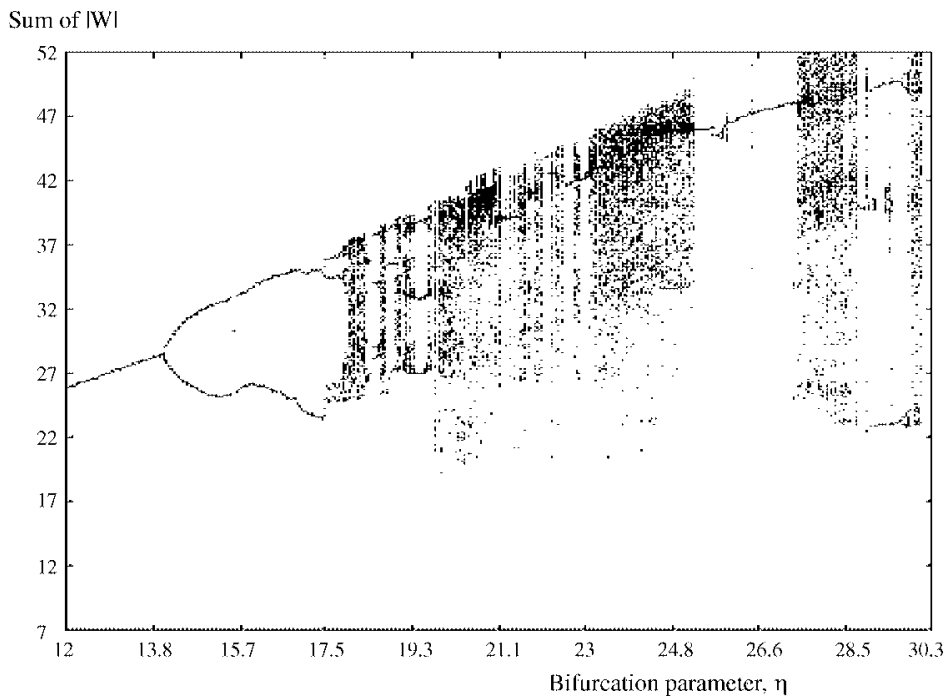

Fig. 5. Bifurcation and the chaos diagram for NN with two nonlinear functions: bipolar sigmoidal of a neuron and $N$-shaped for synaptic coupling. There are $\Delta x=0.0001$ and $\Delta \eta=0.03$.

It should be noted that the fuzziness expressed by input data and weight memberships in the rates given did not influence to the appearence of chaotic phenomena in such simplified neural networks. We intend to continue the simulation experiment of ANN with fuzziness. 


\section{Conclusions}

1. The origin of the fuzzy signals lies in complex biochemical and electrical processes of the synapse and dendrite membrane excitation and the inhibition mechanism.

2. The mathematical operations incorporated in fuzzy neural network modeling are: the scalar product between inputs of layers and synaptic weights is replaced by a fuzzy logic multiplication, the sum of products changes to the fuzzy logic sums, the operators such as supremum, maximum, and minimum are presented for a fuzzy description.

3. The algorithm of varying membership functions built basing on the backpropagation paradigm and the method of fuzzy neural optimization have been considered. Both the fuzzy properties and the chaos phenomenon are analyzed on the basis of experimental computations.

4. We hope that this modest study on fuzziness and chaos phenomena will further stimulate the development of more powerful tools for modeling uncertainties associated with human perception, cognition, and thinking.

\section{References}

Chapeau-Blondeau F., and G. Chauvet (1992). Stable, oscillatory, and chaotic regimes in the dynamics of small neural network with delay, Neural Networks, 5, 735-743.

Cohen M. A., and S. Grossberg (1983). Absolute stability of global pattern formation and parallel memory storage by competitive neural networks, IEEE Trans. SMC, 13(5), 815-826.

Fukai T., and M. Shiino (1990). Asymmetric neural networks incorporating the Dale hypothesis and noisedriven chaos, Physical Review Letters, 64, 1465-1468.

Garliauskas A. (1998) Neural network chaos analysis. Nonlinear Analysis and Control, Vilnius, IMI, 3, $43-57$.

Garliauskas A. (1998). Numerical simulation of dynamic synapse-dendrite-soma neuronal processes, Informatica, 9(2), 141-160.

Garliauskas A., and R. Andzius (1998). Chaotic phenomena in neural networks with nonlinear synaptic functions, In The Proc. of Ist Copenhagen Symposium on Computer Simulation in Biology, Ecology and Medicine, Copenhagen. pp. 81-85.

Garliauskas A., and S. Minkevicius (1998). On stochastic discrete neuronetworks, Informatica, 4(1-2), 140147.

Garliauskas A., and M.J. Shaw (1997). Neural network chaos theory and aplications in finance, In Proc. of the 6th International Workshop on Parallel Applications in Statistics and Economics, Univ. of Economics, Prague. pp. 45-54.

Gupta M.M. (1989). Fuzzy neural network in computer vision. In International Joint Conference on Neural Network, Washington.

Gupta M.M. (1991). Fuzzy neural computing systems. Neural Network World, 2(6), 629-648.

Gupta M.M., and D.H. Rao (1994). On the priciples of fuzzy neural networks, Fuzzy Sets and Systems, 61, $1-18$.

Kaufmann A., M.M. Gupta (1985). Introduction to Fuzzy Arithmetic: Theory and Applications. Van Nostrand Reinhold, New York, Second Edition.

Matsumoto K., and I. Tsuda (1987). Extended information in onedimensional maps, Physica D, 26, 347-357.

Matsumoto K., and I. Tsuda (1988). Calculation of information flow rate from mutual information, Journal of Physics A: Mathematical and General, 64, 3561-3566.

Tsuda I. (1995). Dynamic link of memory - chaotic memory map in nonequillibrium neural networks, Neural Networks, 5, 313-326. 
van der Maas H.L.J., P.F.M.J. Verschure and P.C.M. Molenaar (1990). A note on chaotic behavior in simple neural networks, Neural Networks, 3, 119-122.

Zadeh L.A. (1965). Fuzzy sets, Information and Control, 8, 338-353.

Zadeh L.A. (1968). Fuzzy algorithms, Information and Control, 12, 99-102.

Zadeh L.A., R.E. Bellman (1970). Decision-making in a fuzzy environment, Management Science, 17(4), 141164.

Zimmermann H. J. (1991). Fuzzy Set Theory and Its Applications, Dordrecht, Kluver Academic Press.

A. Garliauskas received his Habil. Dr. degree of technical sciences from the Computer Center, the Department of the USSR Academy of Sciences, Novosibirsk, USSR, in 1977. $\mathrm{He}$ is a head of the Laboratory of Neuroinformatics, Institute of Mathematics and Informatics and a professor in Vilnius Gediminas Technical University. His research interest includes neuroinformatics methodology, control problems and development of neural networks algorithms, chaos processes. 


\section{Dirbtiniai neuromodeliai su neryškumo ir chaoso savybėmis}

\section{Algis GARLIAUSKAS}

Nagrinejjamas apibendrintas neurotinklų modelis atsižvelgiant ic parametrų neryškumą (neapibrèžtuma) ir chaosa. Neryškumas susietas su sinapsiu biocheminiais procesais ir dendritu membranu mechanizmu atsitiktiniais reiškiniais bei galimais iejjimu signalu neapibrěžtumais. Pateikti neryškios logikos algoritmai dirbtinio neurotinklo plotmejje bei chaoso interpretacijoje, duoti skaitinio chaoso pasireiškimo eksperimento rezultatai. 